\title{
What's the Excitement about Excitatory Amino Acids in Amyotrophic Lateral Sclerosis?
}

In this issue, one review and two original articles appear that address the potential role of excitatory amino acids in the pathogenesis of amyotrophic lateral sclerosis (ALS) [1-3]. The hypothesis, as put forward by Plaitakis [1], is that a generalized defect in excitatory amino acid metabolism in ALS may render select groups of neurons vulnerable to excitatory amino acid-induced neurotoxic damage because of their unique neuronal properties and the neurochemical character of their connections. Several studies are cited as providing evidence for increased plasma and cerebrospinal fluid (CSF) glutamate concentrations in patients with ALS, and also decreased concentrations of brain glutamate and decreased spinal cord glutamate, aspartate, $\mathrm{N}$-acetylaspartate (NAA), and $\mathrm{N}$-acetylaspartyl glutamate (NAAG). Plaitakis concludes that the data support the notion of a generalized defect in glutamate metabolism in ALS rather than a selective defect in neuronal glutamate transport, metabolism, storage, release, or receptors. Why, then, is ALS characterized by such regionally specific pathological findings? Would not other regions of the nervous system be affected in ALS if glutamate (which is a neurotransmitter and a potential neurotoxin throughout brain) is metabolized abnormally? Plaitakis argues that the selective vulnerability may reside in the particular properties of motor neuron innervation or factors that could abnormally potentiate this innervation, or both. He suggests that one such potentiator could be glycine. Glycine is an inhibitory neurotransmitter of interneurons in spinal cord and brainstem and is uniquely present in high concentrations in these regions. It is also a positive modulator of one subtype of glutamate receptor, the $N$-methyl-D-aspartate (NMDA) receptor. The NMDA receptor is thought to play a crucial role in glutamate-induced neurotoxicity [4]. Glycine enhances glutamate's excitatory actions at the NMDA receptor and promotes recovery of NMDA receptors after glutamate-induced desensitization. Thus, many nervous system regions may be able to compensate for high extracellular glutamate concentrations except in spinal cord and brainstem where glycine enhances glutamate's actions. In support of Plaitakis' hypothesis is the observation that persons develop selective upper and lower motor neuron disorders after exposure to the glutamatergic neurotoxins in Latbyrus sativa and Cycas circinalis [5].

The data of Rothstein and coworkers [3] support Plaitakis' hypothesis by demonstrating 100 to $200 \%$ increases in glutamate and aspartate concentrations in CSF of patients with ALS. CSF NAA and NAAG are also shown to be increased several-fold. In spinal cord of patients with ALS, NAA and NAAG concentrations are reportedly decreased. In contrast, Perry's group [2] found no abnormalities in CSF glutamate and aspartate levels in patients with ALS. Additionally, they found increases in plasma glutamate in patients with ALS, as observed previously by others, but they attributed these changes to age differences between the ALS and control groups.

What factors can account for the differences between these two studies? The patients studied by Rothstein and associates had been symptomatic for half as long as those in Perry and colleagues' study. Perhaps in more advanced disease, CSF glutarnate levels decrease because of the decreased tissue levels of glutamate. Perry and colleagues' patients, however, did not show a trend toward decreased CSF glutamate concentrations with more advanced disease, and Rothstein and associates' data did not suggest a progressive decrease in tissue glutamate levels with advancing disease. Rothstein and colleagues included only patients with both upper and lower motor neuron disease, whereas Perry and coworkers also included those with only lower motor neuron disease. There is some evidence that isolated lower motor neuron disease may have a different origin than ALS [6].

The striking discrepancy, however, between the Rothstein and the Perry studies is the tremendous difference in total glutamate and aspartate concentrations measured in CSF. In controls, Rothstein and coworkers report glutamate concentrations of $2.9 \mu \mathrm{mol} / \mathrm{L}$, whereas Perry's group reports glutamate concentrations of $0.2 \mu \mathrm{mol} / \mathrm{L}$. These values represent a 15 -fold difference in concentration. Even greater differences are observed in aspartate concentrations. Except for glycine and cystine, other amino acid concentrations are similar in the two studies. Perry and associates explain that glutamate concentrations must be measured in CSF that is deproteinized immediately. Otherwise, 
enzymes in CSF will metabolize the amino acids and artificially elevate glutamate and aspartate levels and lower glutamine and asparagine levels. In 1975, Perry's group revised their estimates of normal CSF amino acid levels based on analysis of deproteinized fluid. At that time, they reported the CSF glutamate values for normal adults as $1.8 \mu \mathrm{mol} / \mathrm{L}[7]$. In the current paper by Perry and coworkers, they find normal values onetenth the earlier revised values using seemingly identical methodology. In fact, the Perry group's current values appear to be below the sensitivity of the assay for aspartate and to approach the sensitivity of the assay for glutamate, since only one significant digit is provided and the standard deviations are large. Is it possible that a more sensitive assay might show differences between control and ALS CSF glutamate concentrations? The Perry group's low CSF glutamate and aspartate values "make sense" to the extent that the nervous system would be expected to have low CSF concentrations of excitatory amino acids so that normal neurotransmission would not be disrupted. Furthermore, Perry and colleagues have used the same assay to show that CSF glutamate concentrations are higher in patients with Huntington's disease than in control patients [8]. Which CSF values are correct, those of Rothstein's group or those of Perry's group? Could the sulfasalicylic acid used by Perry's group precipitate glutamate and aspartate with the protein? What happens to exogenously added glutamate in this assay? Rothstein and colleagues did not deproteinize their fluid, but they assayed the CSF of the controls in parallel with that of the ALS patients and found differences. What factors influenced the observed difference between the ALS patients and the controls? Do the CSF enzymes in ALS metabolize amino acids differently than they do in controls? Further experimentation will be necessary to clarify these issues.

Perry and associates' conclusion that plasma glutamate concentrations are not different between ALS patients and controls seems a bit unwarranted given their current and prior data. Although the control plasma glutamate concentrations in this study showed a correlation with advancing age, in a previous study of twice as many controls, these investigators found no correlation in plasma glutamate concentrations with age [8]. It would seem more appropriate to reassess this issue using an age-matched control group prior to concluding that glutamate does not play a role in this disease. This is especially true because Perry's group has demonstrated previously widespread decreases in brain glutamate in ALS [9].

It appears that although the hypothesis is intriguing, considerably more information needs to be gathered prior to concluding that excitatory amino acids play a role in ALS. In addition, more attention needs to be paid to the selective vulnerability issues raised by
Plaitakis. For instance, the hypothesis that glycine acts in specific regions as a potentiator of glutamate's actions is very interesting. Based on the hypothesis, however, one would expect to see pathological findings in the third nerve nuclei, the periaqueductal gray, and other brainstem structures usually not affected in ALS [10]. Likewise, glycine interneurons are not present in motor cortex where pathological findings are prominent. Furthermore, CSF and tissue glycine levels are thought to be high enough to saturate physiologically the glycine modulatory site on the NMDA receptor in most regions of brain [11]. Finally, spinal cord and brainstem have very low densities of NMDA receptors (about a twentieth those observed in hippocampus). These discrepancies deserve further consideration. If a generalized defect in glutamate metabolism does exist in ALS, perhaps it is a proximate cause of selective neuronal vulnerability but not the primary one. If certain groups of nerve cells are not healthy for other reasons, they may become differentially susceptible to excitatory amino acid-induced damage [12]. One potential approach to the issues might be a study of offspring of persons with familial ALS. Glutamate metabolism could be measured prospectively and correlated with the onset of symptoms.

Although the hypothesis that excitatory amino acids play a role in the pathogenesis of ALS remains speculative, this idea is exciting because of its therapeutic implications. Plaitakis has carried out a trial of branched chain amino acids in ALS based on the theory that these amino acids might stimulate glutamate metabolism and thereby decrease brain glutamate levels [13]. A larger study using these amino acids is now in progress (M. B. Bromberg, personal communication). Antagonists of NMDA receptors are now available and dextromethorphan, which blocks NMDA receptorassociated ion channels [4], is being examined therapeutically in ALS (R. Roos, E. Salazar-Grueso, personal communication, 1990). ALS is a tragic disease and it would be a real breakthrough if such therapies proved effective. Nevertheless, clinical trials of preventive therapies are very expensive and labor intensive. Premature initiation of large trials without convincing preliminary data would be a mistake.

Anne B. Young, $M D, P b D$

Department of Neurology

University of Michigan

Ann Arbor, $M I$

\section{References}

1. Plaitakis A. Glutamate dysfunction and selective motor neuron degeneration in amyotrophic lateral sclerosis: an hypothesis. Ann Neurol 1990;28:3-8

2. Perry TL, Krieger C, Hansen S, Eisen A. Amyotrophic lateral 
sclerosis: amino acid levels in plasma and cerebrospinal fluid. Ann Neurol 1990;28:12-17

3. Rothstein JD, Tsai G, Kuncl RW, et al. Abnormal excitatory amino acid metabolism in amyotrophic lateral sclerosis. Ann Neurol 1990;28:18-25

4. Choi DW. Glutamate neurotoxicity and diseases of the nervous system. Neuron 1988;1:623-634

5. Spencer PS, Nunn PB, Hugon J, et al. Guam amyotrophic lateral sclerosis-parkinsonism-dementia linked to a plant excitant neurotoxin. Science 1987;237:517-522

6. Pestronk A, Chaudhry V, Feldman E, et al. Lower motor neuron syndromes defined by patterns of weakness, nerve conduction abnormalities and high titers of antiglycolipid antibodies. Ann Neurol 1990;27:316-326

7. Perry TL, Hansen S, Kennedy J. CSF amino acids and plasmaCSF amino acid tatios in adults. J Neurochem 1975;24:587589

8. Perry TL, Hansen S. What excitotoxin kills striatal neurons in
Huntington's disease? Clues from neurochemical studies. Neurology 1990;40:20-24

9. Perry TL, Hansen S, Jones K. Brain glutamate deficiency in amyotrophic lateral sclerosis. Neurology 1987;37:1845-1848

10. Probst A, Cortes R, Palacios JM. The distribution of glycine receptors in the human brain. A light microscopic autoradiographic study using $\left[{ }^{3} \mathrm{H}\right]$ strychnine. Neuroscience $1986 ; 17: 11-$ 35

11. Kleckner NW, Dingledine R. Requirement for glycine in activation of NMDA-receptors expressed in Xenopus oocytes. Science 1988;241:835-837

12. Novelli A, Reilly JA, Lysko PG, Henneberry RC. Glutamate becomes neurotoxic via the $\mathrm{N}$-methyl-D-aspartate receptor when intracellular energy levels are reduced. Brain Res 1988;451:205-212

13. Plaitakis A, Mandeli J, Smith J, Yahr MD. Pilot trial of branched-chain amino acids in amyotrophic lateral sclerosis. Lancet 1988;1:1015-1018 